\title{
Semidiurnal signal in UT1 due to the influence of tidal gravitation on the triaxial structure of the Earth
}

\author{
Aleksander Brzeziński ${ }^{1,2}$ and Nicole Capitaine ${ }^{3}$ \\ ${ }^{1}$ Warsaw University of Technology, Faculty of Geodesy and Cartography, Warsaw, Poland \\ ${ }^{2}$ Space Research Centre, Polish Academy of Sciences, Warsaw, Poland \\ ${ }^{3}$ SYRTE, Observatoire de Paris, CNRS, UPMC, Paris, France
}

\begin{abstract}
The axial component of Earth rotation, which is conventionally expressed by Universal Time (UT1), contains small physical signals with diurnal and subdiurnal periods. This part of the spectrum is dominated by the tidal effects which are regular and predictable. The largest components express the influence of the gravitationally forced ocean tides with diurnal and semidiurnal periods and amplitudes up to 0.02 milliseconds (ms) in UT1 corresponding to an angular displacement of 0.30 milliarcseconds (mas); see Table 8.3 of the IERS Conventions (IERS, 2003). There are also smaller subdiurnal components (amplitudes up to 0.03 mas), designated as "spin libration" by Chao et al. (1991), due to direct influence of the tidal gravitation on those features of the Earth's density distribution which are expressed by the non-zonal terms of the geopotential. These components are not included in the models recommended by the IERS Conventions, in contrast to the corresponding effect in polar motion (ibid., Table 5.1).

Here we consider in detail the subdiurnal libration in UT1. We derive an analytical solution for the structural model of the Earth consisting of an elastic mantle and a liquid core which are not coupled to each other. The reference solution for the rigid Earth is computed by using the satellite-determined coefficients of geopotential and the recent developments of the tide generating potential (TGP). We arrived to the conclusion that the set of terms with amplitudes exceeding the truncation level of 0.005 mas consists of 11 semidiurnal harmonics due to the influence of the TGP term $u_{22}$ on the equatorial flattening of the Earth expressed by the Stokes coefficients $C_{22}, S_{22}$. There is an excellent agreement between our estimates for the rigid Earth and the amplitudes derived by Wünsch (1991). The only important difference is the term with the tidal code $\nu_{2}$, which seems to be overlooked in the development of Wünsch. Our amplitudes computed for an elastic Earth with liquid core appear to be in reasonable agreement with those derived by Chao et al. (1991), but the latter model was not complete. The estimated effect is superimposed on the ocean tide influences having the same frequencies but 9 to 11 times larger amplitudes. Nevertheless, its maximum peak-to-peak size is about 0.105 mas, hence definitely above the current uncertainty of UT1 determinations. Comparison with the corresponding model of prograde diurnal polar motion associated with the Earth's triaxiality (IERS Conventions, Table 5.1) shows that: 1) the two effects are of similar size, 2) there is consistency between the underlying dynamical models, parameters employed, etc. In conclusion, we recommend adding the model developed here to the set of procedures provided by the IERS Conventions.
\end{abstract}

Keywords. Earth rotation, universal time, tidal gravitation

\section{References}

Brzeziński, A. \& Capitaine, N. 2002, in: N. Capitaine (ed.), Proc. Journees 2001, 51, Obs. de Paris

Chao, B. F., Dong, D. N., Liu, H. S., \& Herring T. A. 1991, Geophys. Res. Letters 18, 2007

IERS 2003, D. McCarthy \& G. Petit (eds.), IERS Technical Note 32, Verlag des Bundesamts für Kartographie und Geodäsie, Frankfurt am Main

Wünsch, J. 1991, Astron. Nachr. 312, 321 